(1E,1'E)-N,N'-(hekzan-1,6-diil)bis(1-(tiyofen-2-il)metanimin) ve (1E,1'E)-N,N'-(oktan-1,8diil)bis(1-(tiyofen-2-il)metanimin) Schiff Baz Ligantlarının Deneysel ve Teorik Karakterizasyonu

\author{
Gühergül ULUÇAM ${ }^{1 *}$, Büşra YENTÜRK ${ }^{1}$
}

ÖZET: Aromatik aldehit olan 2-tiyofen-karboksialdehit sırasıla 1,6-diaminohekzan ve 1,8-diaminooktan'nın kullanılmasiyla (1E,1'E)-N,N'-(hekzan-1,6-diil)bis(1-(tiyofen-2-il)metanimin) ve (1E,1'E)-N,N'-(oktan-1,8diil)bis(1-(tiyofen-2-il)metanimin) Schiff baz ligantları elde edildi. Ligantlar fourier transform infrared spektroskopisi (FT-IR), ${ }^{1} \mathrm{H}$ - ve ${ }^{13} \mathrm{C}$ - nükleer manyetik rezonans spektroskopisi $\left({ }^{1} \mathrm{H}\right.$ - ve ${ }^{13} \mathrm{C}$ - NMR), kütle spektroskopisi (LC ESI/MS) yöntemleri ile karakterize edildi. Ligantların antibakteriyal aktiviteleri araştırıldı. Ayrıca ligantların bağ uzunlukları, bağ açıları, dihedral açıları gibi geometrik özellikleri, elektronik özellikleri, en yüksek dolu moleküler orbital (HOMO) ve en düşük boş moleküler orbital (LUMO) enerjileri Gaussian 09w programı kullanılarak hesaplandı. Deneysel ve teorik spektrum verileri karşılaştırıldı.

Anahtar Kelimeler: Tiyofen, Schiff baz, Antibakteriyal Aktivite, Gaussian 09w, YFT/B3LYP.

\title{
Experimental and Theoretical Analysis of (1E,1'E)-N,N'-(hexane-1,6-diyl)bis(1-(thiophen-2- yl)methanimine) and (1E,1'E)-N,N'-(octane-1,8-diyl)bis(1-(thiophen-2-yl)methanimine) Schiff Base Ligands
}

\begin{abstract}
The aromatic aldehyde thiophene-2-carbaldehyde, using 1,6-diaminohexane and 1,8diaminoctane, respectively (1E,1'E)-N,N'-(hexane-1,6-diyl)bis(1-(thiophen-2-yl)methanimine) and $\left(1 E, 1^{\prime} E\right)$ $N, N^{\prime}$-(octane-1,8-diyl)bis(1-(thiophen-2-yl)methanimine) Schiff base ligands were obtained. The ligands were characterized by fourier transform infrared spectroscopy (FT-IR), ${ }^{1} \mathrm{H}$ - and ${ }^{13} \mathrm{C}$ - nuclear magnetic resonance spectroscopy ( ${ }^{1} \mathrm{H}$ - and ${ }^{13} \mathrm{C}$ - NMR), mass spectroscopy (LC ESI/MS) methods. The antibacterial activity of the ligands was investigated. In addition, the geometric properties of ligands such as bond lengths, bond angles, dihedral angles, electronic properties, highest occupied molecular orbital (HOMO) and lowest unoccupied molecular orbital (LUMO) energies were calculated by using Gaussian 09w program. Experimental and theoretical spectrum data were compared.
\end{abstract}

Keywords: Thiophen, Schiff base, Antibacterial Activity, Gaussian 09w, DFT/B3LYP.

\footnotetext{
${ }^{1}$ Gühergül ULUÇAM (Orcid ID: 0000-0001-6481-8483), Büşra YENTÜRK (Orcid ID: 0000-0003-0009-9752), Trakya Üniversitesi, Fen Fakültesi, Kimya Bölümü, Edirne, TÜRKIYYE

* Sorumlu Yazar / Corresponding Author: Gühergül ULUÇAM, e-mail: absunbul@ beun.edu.tr

* Bu çalışma Büşra YENTÜRK’ün Yüksek Lisans tezinin bir bölümüdür. 


\section{GíRİ̧}

Donör atomlara sahip olan Schiff baz ligantları ve kompleksleri endüstride, klinik ve biyolojik sistemlerde oldukça yaygın kullanım alanlarına sahiptir (Hamada, 1997; Karakaya ve ark., 2016). İlaç, tarım, teknoloji, polimer teknolojisi, elektronik gibi kullanım alanlarının yanı sıra özellikle kanser hücrelerinin tedavisinde önemli çalışmalar yapılmaktadır (Dineshkumar ve Muthusamy, 2016; Kumar ve ark., 2009; Sun ve ark., 2015; Suresh ve ark., 2015). Ayrıca antitümör, antibakteriyel, antiviral, antiparazit gibi etkilerinin olduğu da gösterilmiştir (Fedorova ve ark., 2013; Pessoa ve ark., 2015; Rehder, 2003; Thompson, 1999).

2-tiyofen karbaldehit sirasiyla 1,6diaminohekzan ve 1,8-diaminooktan ile Schiff baz kondenzasyon metoduyla, iki ligant sentezlendi. Ligantların yapisı fourier transform infrared spektroskopisi (FTIR), ${ }^{1} \mathrm{H}$ - ve ${ }^{13} \mathrm{C}$ nükleer manyetik rezonans spektroskopisi $\left({ }^{1} \mathrm{H}-\right.$ ve ${ }^{13} \mathrm{C}$ - NMR), kütle spektroskopisi (LC ESI/MS) ile aydınlatılarak, antibakteriyal aktiviteleri araştırıldı. Teorik özellikleri, B3LYP/6-311G++(2d,p) temel setleri yoğunluk fonksiyonel teorisi (YFT) kullanılarak incelendi. $\mathrm{Bu}$ baz setinin seçimi ile elde edilen teorik sonuçların deneysel sonuçlarla uyumluluğunu yeterince hassaiyetle sağlanmıştır. Gaussian G09w paket program kullanılarak NMR ve FTIR değerleri hesaplandı. Bağ uzunlukları, bağ ve dihedral açılar, moleküler elektrostatik potansiyel haritası (MEP), dipol momentleri, en yüksek dolu moleküler orbital enerjisi (HOMO) ve en düşük boş moleküler orbital enerjisi (LUMO) saptand. Ligantları hangi konformasyonda bulunacağı yapılan teorik hesaplamalar ile belirlendi (Nagy, 2013).

\section{MATERYEL VE YÖNTEM}

FTIR spektrumu için $450-4000 \mathrm{~cm}^{-1}$ aralığında Perkin-Elmer BXII spektrometresi kullanıldı. ${ }^{1} \mathrm{H}$ - ve ${ }^{13} \mathrm{C}$ - NMR spektrumları, sirasıyla Varian $300 \mathrm{MHz}$ ve Varian $75.5 \mathrm{MHz}$ de dötero kloroform $\left(\mathrm{CDCl}_{3}\right)$ çözücüsü kullanılarak alındı. Kütle spektrumları Agilent Technologies 6460 Triple Quad LC ESI/MS ile elde edildi. Antibakteriyal aktivitelerin belirlenmesinde absorbans ölçümleri için Thermoscientific Multiskan Go multiplate spektrofotometresi kullanıld.

\section{Schiff Baz Ligantlarm Eldesi}

1,6-diaminohekzan $(0.58 \mathrm{~g}, 5 \mathrm{mmol})$ ve 2 tiyofen karbaldehit $(1.12 \mathrm{~g}, \quad 10 \mathrm{mmol}) \quad 50 \mathrm{ml}$ metanol bulunan iki ayrı balonda çözüldü. İki çözelti bir saat süreyle karıştırılırken 1-2 damla glasiyal asetik asit damlatıldı. Tepkime iki gün 1sıtılarak devam ettirildi. Daha sonra çözücü uzaklaştırıldı. Kalan madde, hekzan:kloroform (20:10ml) içinde bekletildi ve vakum etüvünde kurutuldu. Koyu sarı renkli viskos ligant (1E,1'E)-N,N'-(hekzan-1,6-diil)bis(1-(tiyofen-2il)metanimin) (A) sentezlendi (Casella ve Ibers, 1981; Mihai ve ark., 2009). Stokiyometrik oranları aynı aldehit ile 1,8-diaminooktan (0.72g, $5 \mathrm{mmol})$ kullanılarak deney prosedürü tekrar uygulandı. Koyu sarı renkli viskos ligant $\left(1 E, 1^{\prime} E\right)$ - $N, N^{\prime}$-(oktan-1,8-diil)bis(1-(tiyofen-2il)metanimin) (B) sentezlendi. A ve B ligantlarının elde reaksiyonu Şekil 1. de gösterildi. 


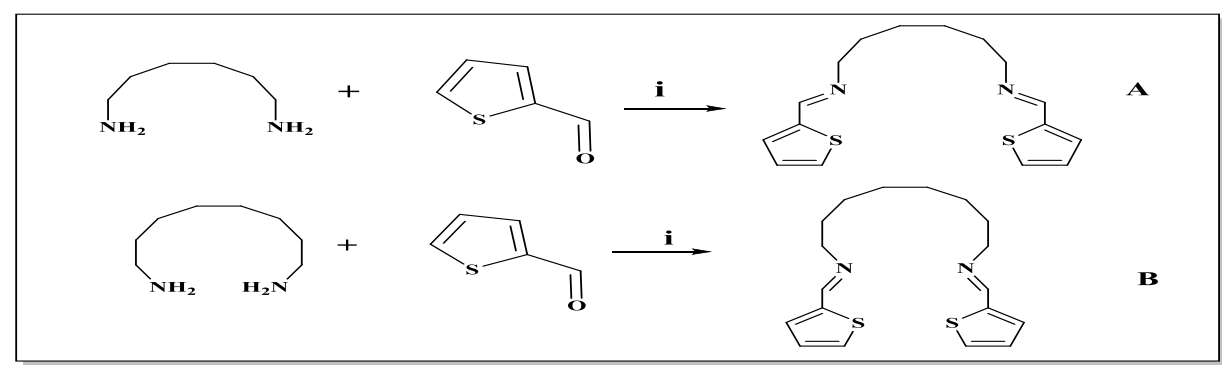

Şekil 1. A ve B ligantlarının elde reaksiyonu (i:metanol, glasiyal asetik asit)

(A):Yield: $1.13 \mathrm{~g}(73 \%)$, FT-IR $\left(\mathbf{c m}^{-1}\right)$ : 30702853, 1632, 1535, 1345, 755. $\boldsymbol{\delta}_{\mathbf{H}}(\mathbf{3 0 0}$ MHz; CDCl3): $1.53-1.38$ (4H, m, CH2), 1.64-1.68 $(4 \mathrm{H}, \mathrm{m}, \mathrm{CH} 2), 3.52(4 \mathrm{H}, \mathrm{t}, \mathrm{CH} 2), 6.99-7.01(2 \mathrm{H}$, $\mathrm{m}, \mathrm{CH}), 7.25(2 \mathrm{H}, \mathrm{d}, \mathrm{CH}), 7.31(2 \mathrm{H}, \mathrm{d}, \mathrm{CH})$, $8.29(2 \mathrm{H}, \mathrm{s}, \mathrm{CHN}) . \boldsymbol{\delta}_{\mathbf{C}}(\mathbf{7 5 . 5} \mathbf{M H z} ; \mathbf{C D C l 3})$ : $27.31(\mathrm{CH} 2), 30.99(\mathrm{CH} 2), 61.50(\mathrm{CH} 2), 127.59$, 128.80, $130.45(\mathrm{CH}), 142.78(\mathrm{C}), 154.28(\mathrm{CHN})$. ESI/MS (m/z) $[\mathrm{M}+\mathrm{H}]^{+},(305.1000)$.

(B):Yield: $1.18 \mathrm{~g}(71 \%)$, FT-IR $\left(\mathbf{c m}^{-1}\right)$ : 3071 2925, 1633, 1524, 1346, 756. $\boldsymbol{\delta}_{\mathbf{H}}(\mathbf{3 0 0} \mathbf{~ M H z ; ~}$ CDCl3): $1.23-1.25$ (8H, m, CH2), 1.58-1.62 (4H, m, CH2), 3.44 (4H, t, CH2), 6.91-6.94 (2H, $\mathrm{m}, \mathrm{CH}), 7.16(2 \mathrm{H}, \mathrm{d}, \mathrm{CH}), 7.23(2 \mathrm{H}, \mathrm{d}, \mathrm{CH})$, $8.21(2 \mathrm{H}, \mathrm{s}, \mathrm{CHN}) . \boldsymbol{\delta}_{\mathrm{C}}(\mathbf{7 5 . 5} \mathrm{MHz} ; \mathbf{C D C l})$ : 27.49 (CH2), $29.57(\mathrm{CH} 2), 31.06\left(\mathrm{CH}_{2}\right), 61.53$ $(\mathrm{CH} 2), 127.54,128.75,130.38(\mathrm{CH}), 142.79(\mathrm{C})$, $154.12 \quad(\mathrm{CHN}) . \quad$ ESI/MS $(\mathbf{m} / \mathbf{z}) \quad[\mathrm{M}+\mathrm{H}]^{+}$, (333.1000).

\section{Antibakteriyal Aktivite}

Klinik Laboratuar Standartlar Enstitüsü (CLSI) tarafindan önerilen Besiyeri MikroSeyreltme (Broth Microdilution) yöntemi kullanıld1. Escherichia coli ATCC 25922, Staphylococcus aureus (Rosenbach, 1884) ATCC 25923, Listeria monocytogenes ATCC 19115, Salmonella thphimurium (Lignieres, 1900) ATCC 14028, Bacillus cereus (Frankland \& Frankland, 1887) ATCC 11778 bakterileri 24 saat $37^{\circ} \mathrm{C}$ de Tryptic Soy Broth besiyerinde inkübe edildi. McFarland Skalası da 0.5 'e ayarlandı. Antibiyotik kontrolü olarak Ampisilin kullanıldı. Sterilite amaciyla antibiyotikler ve çözünen madde stok çözeltileri $0.45 \mu \mathrm{m}$ steril filtreden süzüldü. Ligantlar, $200 \mu \mathrm{g} / \mathrm{ml}$ 'den başlayarak yarı yarıya seyreltilerek, altı doz olacak şekilde ayarlandı. 96 lik plakanın her bir kuyusuna, besiyerinden $150 \mu \mathrm{L}$, bakteri kültürlerinden $30 \mu \mathrm{L}$ ve liganttan $20 \mu \mathrm{L}$ ekildi. Sterilite kontrolü olarak sadece madde eklenmiş, besiyeri büyüme kontrolü olarak sadece mikroorganizma ekilmiş besiyeri kullanıldı. Bütün mikroplakalar 24 saat $37^{\circ} \mathrm{C}$ de inkübe edildi ve $600 \mathrm{~nm}$ 'de absorbans değerleri ölçüldü (Ulucam Guhergul, 2017).

\section{Teorik Metot}

Gaussian G09w paket programında Becke'nin üç parametreli hibrid değişen fonksiyoneli ve Lee-Yang-Parr'in korelasyon fonksiyonelinden oluşan B3LYP teorisi kullanılarak 6-311++G(2d,p) temel seti ile hesapland1 (Sreedasyam ve ark., 2013; Turkyilmaz ve ark., 2017; Ulucam G. ve Turkyilmaz, 2018). NMR ve FTIR spektraları, aynı metod ve temel set kullanılarak optimize edilmiş geometrilerden hesaplandı. GIAO metodu, teorik ${ }^{1} \mathrm{H}-$ ve ${ }^{13} \mathrm{C}$ - NMR kimyasal kaymalarının elde edilmesinde kullanıldı. FTIR spektrumu, teorik hataları gidermek için 0.9613 değeri ile çarpılarak düzeltildi (Al-Otaibi ve AlWabli, 2015; Uluçam ve ark., 2015). Ligantların bağ uzunlukları, bağ ve dihedral açıları, HOMOLUMO enerjileri YFT ve 6-311++G(2d,p) temel seti kullanılarak hesapland. Moleküler elektrostatik potansiyel haritası (MEP) gösterildi. 


\section{BULGULAR VE TARTIŞMA}

\section{Moleküler Geometrisi}

A ve B'nin minimize enerjiye sahip üç boyutlu geometrisi GaussView programında çizilmiştir. Geometriler Şekil 2'de gösterilmektedir. Moleküler yapılarda karbonlara bağlı hidrojenler, spektrum verilerinde bağlı oldukları karbon atomlarının numaraları ile temsil edilir. Ligantların atomları arasındaki bağ uzunlukları Çizelge I'de, önemli bağ ve dihedral açıları ise Çizelge II ile verilmektedir. Ligantlar, simetrik bir yapıya sahip olduğundan, bu simetrinin tek bir parçasına ait veriler çizelgelerde yer almaktadır.
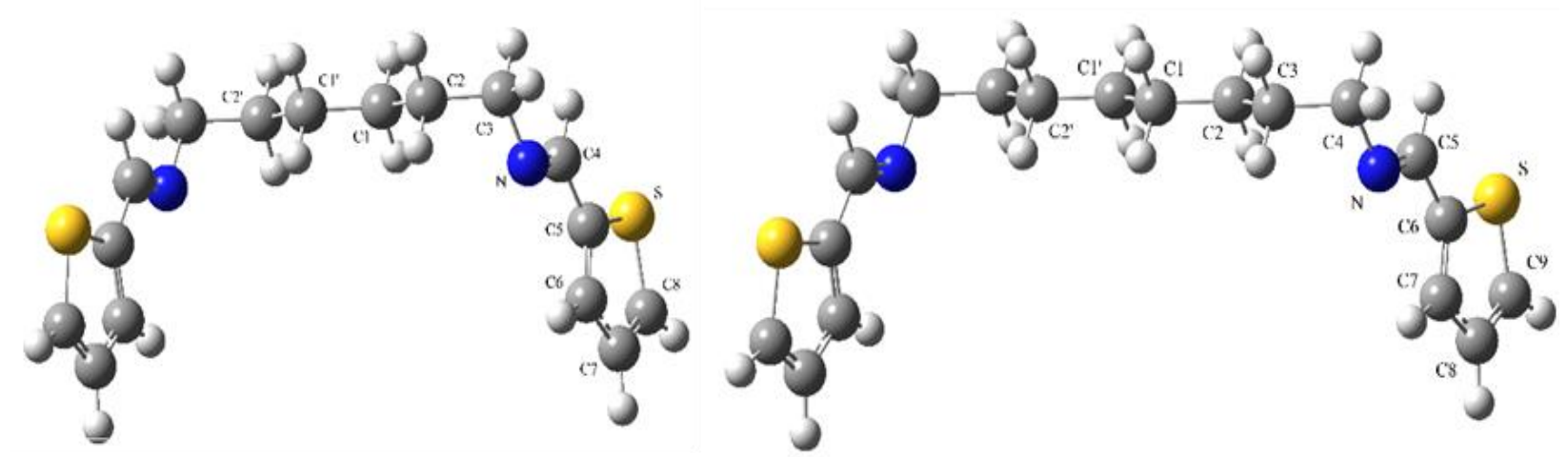

Şekil 2. A ve B ligantlarının moleküler konfigürasyonu (sol:A, sağ:B)

Çizelge 1. A ve B ligantlarının bağları arasındaki teorik uzaklıklar

\begin{tabular}{cccc|cccc}
\hline \multicolumn{3}{c}{ A } & \multicolumn{3}{c}{ B } \\
\hline \multicolumn{3}{c}{ Bă̆ Uzunlukları $(\AA)$} & & \multicolumn{3}{c}{ Bă Uzunlukları $(\AA)$} \\
C1-C2 & 1.529 & C8-C7 & 1.368 & C1-C2 & 1.530 & C9-C8 & 1.368 \\
C2-C3 & 1.532 & C7-C6 & 1.415 & C2-C3 & 1.529 & C8-C7 & 1.415 \\
C3-N & 1.451 & C6-C5 & 1.373 & C3-C4 & 1.532 & C7-C6 & 1.373 \\
N-C4 & 1.268 & C4-H4 & 1.100 & C4-N & 1.451 & C5-H5 & 1.100 \\
C4-C5 & 1.456 & C3-H3x & 1.102 & N-C5 & 1.268 & C4-H4x & 1.102 \\
C5-S & 1.742 & C3-H3y & 1.095 & C5-C6 & 1.456 & C4-H4y & 1.095 \\
S-C8 & 1.721 & & & C6-S & 1.742 & & \\
& & & & S-C9 & 1.721 & & \\
\hline
\end{tabular}

Çizelge 2. A ve B ligantlarının atomları arasındaki teorik bağ açıları ve dihedral açılar

\begin{tabular}{|c|c|c|c|c|c|c|c|}
\hline \multicolumn{4}{|c|}{$\mathbf{A}$} & \multicolumn{4}{|c|}{$\bar{B}$} \\
\hline \multicolumn{2}{|c|}{ Bağ Açıları ( $\left.{ }^{\circ}\right)$} & \multicolumn{2}{|c|}{ Dihedral Açılar( $\left(^{\circ}\right)$} & \multicolumn{2}{|c|}{ Băg Açıları ( $\left.{ }^{\circ}\right)$} & \multicolumn{2}{|c|}{ Dihedral Açılar( $\left(^{\circ}\right)$} \\
\hline $\mathrm{C} 1-\mathrm{C} 2-\mathrm{C} 3$ & 113.962 & $\mathrm{C} 1-\mathrm{C} 2-\mathrm{C} 3-\mathrm{N}$ & -66.110 & C1-C2-C3 & 113.265 & C1-C2-C3-C4 & -179.151 \\
\hline $\mathrm{C} 2-\mathrm{C} 3-\mathrm{C} 4$ & 111.702 & $\mathrm{C} 3-\mathrm{N}-\mathrm{C} 4-\mathrm{C} 5$ & 179.647 & $\mathrm{C} 2-\mathrm{C} 3-\mathrm{C} 4$ & 113.952 & $\mathrm{C} 2-\mathrm{C} 3-\mathrm{C} 4-\mathrm{N}$ & -66.499 \\
\hline C3-N-C4 & 118.559 & C5-S-C8-C7 & 0.0154 & $\mathrm{C} 4-\mathrm{C} 5-\mathrm{N}$ & 111.679 & C4-N-C5-C6 & 179.652 \\
\hline N-C4-C5 & 122.522 & C7-C6-C5-C4 & 179.894 & C4-N-C5 & 118.567 & C6-S-C9-C8 & -0.0259 \\
\hline $\mathrm{C} 5-\mathrm{S}-\mathrm{C} 8$ & 91.800 & & & $\mathrm{~N}-\mathrm{C} 5-\mathrm{C} 6$ & 122.522 & C8-C7-C6-C5 & 179.869 \\
\hline S-C8-C7 & 111.598 & & & C6-S-C9 & 91.798 & & \\
\hline C7-C6-C5 & 113.173 & & & S-C9-C8 & 111.604 & & \\
\hline
\end{tabular}


A ve B'nin bağ uzunlukları, bağ açıları ve dihedral açıları birbiri ile çok uyumludur. A ligantından fazla olarak B ligantının köprüdeki metil fazlalı̆̆ 1 , teorik hesaplamalara göre iki ligantın geometrik parametreleri arasında önemli bir farklılık oluşturmamaktadır.

\section{HOMO-LUMO Analizleri ve Elektronik Özellikleri}

Frontier moleküler orbitalleri (FMO), elektronik ve optik özellik gibi bir moleküle ait özelliklerin saptanmasında önemlidir. Molekül hakkında bilgi sahibi olmak için HOMO-LUMO arasındaki enerji farkı kullanılabilir. HOMO enerjisi, Iyonizasyon Potansiyeli (IP) ile LUMO enerjisi Elektron Afinitesi (EA) ile ilgilidir
(Tamer ve ark., 2016). HOMO-LUMO arasındaki enerji farkı ne kadar az ise molekül o kadar polarize olabilir. Enerji farkı büyük ise reaksiyon verme yeteneğinin düşük olduğu söylenebilir. Polarize olabilen ve HOMOLUMO arasındaki enerji farkı düşük olan moleküller yumuşak molekül olarak tanımlanırken, enerji farkı büyük moleküller ise sert moleküller olarak adlandırılırlar. B3LYP teorisi ve 6-311++G(2d,p) temel seti kullanılarak hesaplanan ligantlarının HOMO-LUMO enerji farkı Şekil 3.'de gösterildi. Atomik birimler (a.u.), elektrostatik birimlere dönüştürüldü (1a.u. = 27.2116 eV) (Vela ve Gazquez, 1990).
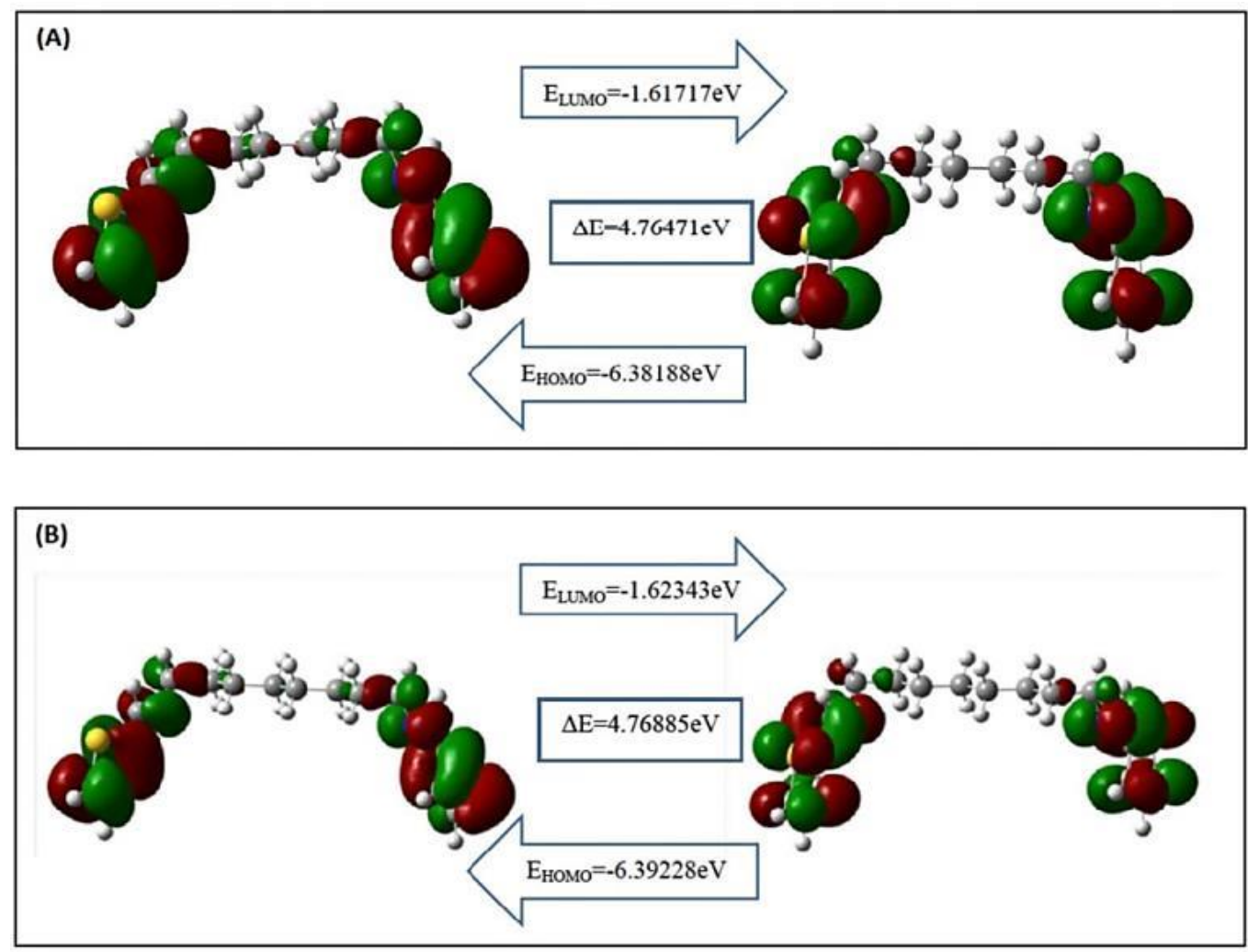

Şekil 3. A ve B ligantlarının işgal edilmiş ve işgal edilmemiş moleküler orbitallerinin üç boyutlu gösterimi ve Enerji değerleri

FMO enerjilerinden, elektronegatiflik, kimyasal potansiyel, kimyasal sertlik ve kimyasal yumuşaklık hesaplandı (Altürk ve ark., 2018; Vela ve Gazquez, 1990). Moleküler elektriksel özellikler, HOMO ve LUMO enerjileri kullanılarak elde edilip Çizelge III'de verildi. 
Çizelge 3. A ve B ligantlarının elektronik yapı değerleri

\begin{tabular}{ccc}
\hline & A & B \\
\hline $\mathrm{E}_{\text {HOMO }}(\mathrm{eV})$ & -6.381888 & -6.392287 \\
$\mathrm{E}_{\text {LUMO }}(\mathrm{eV})$ & -1.617173 & -1.623431 \\
$\mathrm{E}=\mathrm{E}_{\mathrm{LUMO}}-\mathrm{E}_{\mathrm{HOMO}}(\mathrm{eV})$ & 4.764715 & 4.768855 \\
$\mathrm{IP}(\mathrm{eV})$ & 6.381888 & 6.392287 \\
$\mathrm{EA}(\mathrm{eV})$ & 1.617173 & 1.623431 \\
$\chi(\mathrm{eV})$ & 3.999530 & 4.007859 \\
$\varphi(\mathrm{eV})$ & -3.999530 & -4.007859 \\
$\eta(\mathrm{eV})$ & 2.382357 & 2.384428 \\
$\sigma\left(\mathrm{eV}^{-1}\right)$ & 0.419752 & 0.419387 \\
\hline
\end{tabular}

$\mathrm{IP}=$ İyonizasyon Potansiyeli $=-\mathrm{HOMO}, \mathrm{EA}=$ Elektron Afinitesi $=$-LUMO

$\chi=$ Elektronegatiflik $=(\mathrm{IP}+\mathrm{EA}) / 2, \varphi=$ Kimyasal Potansiyel $=-\chi$

$\eta=$ Kimyasal Sertlik $=($ IP-EA $) / 2, \sigma=$ Kimyasal Yumuşaklık $=1 / \eta$

\section{Moleküler Elektrostatik Potansiyel Yüzey}

Ligantlara ait pozitif ve negatif bölgelerinin gösterilmesinde MEP kullanılır. Buluta benzeyen bu harita, liganttaki reaktif bölgelerin kalitatif olarak incelenmesini sağlar. Haritada kırmızı bölgeler kısmi negatif yükü, mavi bölgeler ise kısmi pozitif yükü ifade eder. Daha az elektron içeren bölgeler ise sarı ile gösterilirken hemen hemen nötral bölgeler yeşil ile gösterilir. MEP, moleküler yapı ile biyomoleküller ve ilaçlar dahil olmak üzere moleküllerin fizyokimyasal ilişkisi arasındaki bağlantıyı incelemek için çok yararlı bir araç olduğu bulunmuştur (Alkorta ve Perez, 1996; Gadre ve Shrivastava, 1991; Luque ve ark., 1993; Murray ve Sen, 1996).
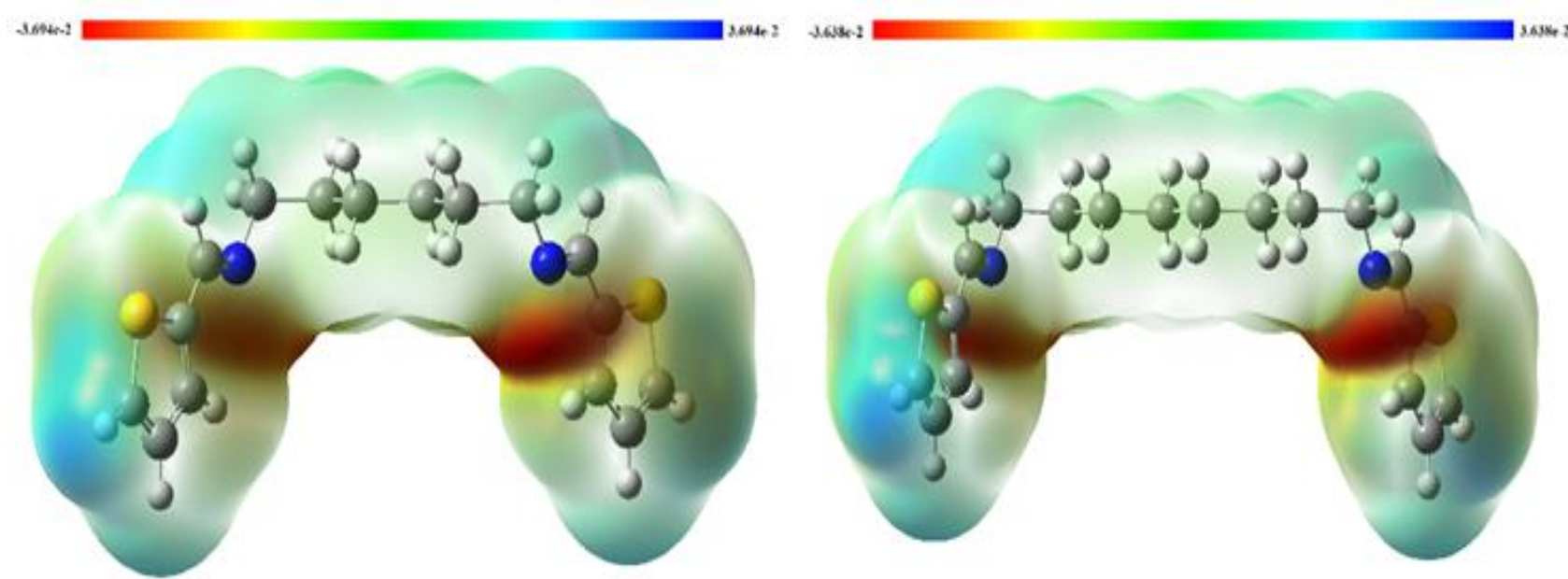

Şekil 4. A ve B ligantlarının moleküler elektrostatik potansiyel haritası (sol:A, sağ:B)

Ligantların MEP haritası hesaplandı. Şekil 4'deki gibi imin grubu azotunun bulunduğu bölge kırmızı ve beşli halkadaki kükürtün bulunduğu bölge sarı gözükmektedir. Kırmızı bölge elektronca zengin bölge olup nükleofilik bölge olarak tanımlanabilir. Ligantların büyük bir kısmını kaplayan yeşil renkli bölgeler metil gruplarının bulunduğu bölgeye denk gelmektedir ve nükleofillerin ilgi duyacağı bölgedir. 


\section{Deneysel ve Teorik ${ }^{13} \mathrm{C}$-NMR ve ${ }^{1} \mathrm{H}-\mathrm{NMR}$} Kimyasal Kayma Değerleri

A'nın ${ }^{13} \mathrm{C}$-NMR spekturumuna göre sirasiyla $\mathrm{CH}_{2}$ karbonları 27.31, 30.99 ve 61.50 ppm de, aromatik ve HCN karbonları 127.59, 128.80, 130.45, 142.78, 154.28 ppm'de gözlendi. B'nin ${ }^{13} \mathrm{C}$-NMR spekturumuna göre sirasiyla CH2 karbonları 27.49, 29.57, 31.06 ve $61.53 \mathrm{ppm}$ de, aromatik ve HCN karbonları 127.54, 128.75, 130.38, 142.79, 154.12 ppm'de gözlendi. A'nın ${ }^{1} \mathrm{H}-\mathrm{NMR}$ spektrumuna göre CH2'ye ait pikler $1.38-1.53,1.64-1.68$ ve 3.52 ppm de, aromatik hidrojenlere ait pikler 6.99-
7.01, 7.25 ve 7.31ppm de, CHN'e ait pik ise 8.29ppm'de bulundu. B'nin ${ }^{1} \mathrm{H}-\mathrm{NMR}$ spektrumuna göre $\mathrm{CH} 2$ ye ait pikler 1.23-1.25, $1.58-1.62$ ve $3.44 \mathrm{ppm}$ de, aromatik hidrojenlere ait pikler 6.91-6.94, 7.13 ve $7.23 \mathrm{ppm}$ de, CHN'e ait pik ise $8.21 \mathrm{ppm}$ 'de bulundu. Ligantlar için öncelikle deneysel olarak gözlenen kimyasal kayma değerleri verilerine göre, ${ }^{1} \mathrm{H}-\mathrm{NMR}$ spektrumlarındaki proton integrasyonları, sentezlenen yapıdaki proton integrasyonuna eşit ve ${ }^{13} \mathrm{C}-\mathrm{NMR}$ spekturumlarındaki karbon sayısının da uygun olduğu görüldü.

Çizelge 4. A ve B ligantlarının TMS'ye göre ${ }^{13} \mathrm{C}-\mathrm{NMR}$ deneysel ve teorik kimyasal kayma değerleri $\delta / \mathrm{ppm}$ (simetrik karbonlar parantez içinde verilmiştir)

\begin{tabular}{rrr|rrr}
\hline & A & & \multicolumn{3}{c}{ B } \\
\hline C Num. & Teorik & Deneysel & C Num. & Teorik & Deneysel \\
\hline C1(C1') & 32.795 & 27.31 & C2(C2') & 32.939 & 27.49 \\
C2(C2') & 35.188 & 30.99 & C3(C3') & 35.402 & 29.57 \\
C3(C3') & 67.829 & 61.50 & C1(C1') & 36.189 & 31.06 \\
C6(C6') & 128.568 & 127.59 & C4(C4') & 67.948 & 61.53 \\
C7(C7') & 131.469 & 128.80 & C7(C7') & 128.518 & 127.54 \\
C8(C8') & 136.622 & 120.45 & C8(C8') & 132.032 & 128.75 \\
C5(C5') & 153.466 & 142.78 & C9(C9') & 136.952 & 130.38 \\
C4(C4') & 154.921 & 154.28 & C6(C6') & 154.245 & 142.28 \\
& & & C5(C5') & 156.603 & 154.12
\end{tabular}

TMS: Tetrametil silan, Karbon Numarası: C Num.

A ve $\mathbf{B}$ için ${ }^{13} \mathrm{C}-\mathrm{NMR}$ ve ${ }^{1} \mathrm{H}-\mathrm{NMR}$ kimyasal kayma değerleri, gaz fazında 6$311++\mathrm{G}(2 \mathrm{~d}, \mathrm{p})$ metodu kullanılarak hesapland (Çizelge IV ve V). A için C1(C1'), C2(C2') ve C3(C3') karbonlar1 32.795, 35.188 ve 67.829ppm'de, C6(C6'), C7(C7'), C8(C8'), C5(C5'), C4(C4') karbonları sirasiyla, 128.568, $131.469,136.622,153.466$ ve $154.921 \mathrm{ppm}$ 'de hesapland1. B için C2(C2'), C3(C3'), C1(C1') ve C4(C4') karbonlar1, 32.939, 35.402, 36.189 ve 67.948ppm'de, C7(C7'), C8(C8'), C9(C9'), C6(C6'), C5(C5') karbonlar sirasiyla 128.518, $132.032,136.952,154.245$ ve $156.603 p p m$ 'de hesapland1. A için H1x(H1x'), H1y(H1y'),
H2x(H2x'), H2y(H2y'), H3x(H3x'), H3y(H3y') protonlar1, $0.818,1.193,1.459,1.943,3.046$ ve 4.256ppm'de, H7(H7'), H8(H8'), H6(H6'), H4(H4') protonları sirasiyla 7.161, 7.519, 8.064 ve $8.425 \mathrm{ppm}$ 'de hesapland1. B için $\mathrm{H} 2 \mathrm{x}\left(\mathrm{H} 2 \mathrm{x}^{\prime}\right)$, H1x(H1x'), H1y(H1y'), H2y(H2y'), H3x(H3x'), H3y(H3y'), H4x(H4x'), H4y(H4y') protonlar1 sirasiyla, 0.771, 1.108, 1.159, 1.219, 1.472, 1.93, 3.079 ve $4.215 \mathrm{ppm}$ 'de, H8(H8'), H9(H9'), H7(H7'), H5(H5') protonlar1 sirasiyla 7.182, $7.510,8.066$ ve $8.466 \mathrm{ppm}$ 'de hesapland1. Teorik ve deneysel NMR spektrum değerleri karşılaştırıldığında, aralarındaki uyum açıkça gözükmektedir. 
Çizelge 5: A ve B ligantlarının TMS'ye göre ${ }^{1} \mathrm{H}-\mathrm{NMR}$ deneysel ve teorik kimyasal kayma değerleri $\delta / \mathrm{ppm}$ (simetrik protonlar parantez içinde verilmiştir.)

\begin{tabular}{|c|c|c|c|c|c|}
\hline \multicolumn{3}{|c|}{$\mathbf{A}$} & \multicolumn{3}{|c|}{ B } \\
\hline H Num. & Teorik & Deneysel & H Num. & Teorik & Deneysel \\
\hline H1x(H1x') & 0.818 & $1.38-1.53$ & $\mathrm{H} 2 \mathrm{x}\left(\mathrm{H} 2 \mathrm{x}^{\prime}\right)$ & 0.771 & $1.23-1.25$ \\
\hline H1y(H1y') & 1.193 & & $\mathrm{H} 1 \mathrm{x}\left(\mathrm{H} 1 \mathrm{x}^{\prime}\right)$ & 1.108 & \\
\hline $\mathrm{H} 2 \mathrm{x}\left(\mathrm{H} 2 \mathrm{x}^{\prime}\right)$ & 1.459 & $1.64-1.68$ & H1y(H1y') & 1.159 & \\
\hline H2y(H2y') & 1.943 & & $\mathrm{H} 2 \mathrm{y}\left(\mathrm{H} 2 \mathrm{y}^{\prime}\right)$ & 1.219 & \\
\hline $\mathrm{H} 3 \mathrm{x}\left(\mathrm{H} 3 \mathrm{x}^{\prime}\right)$ & 3.046 & 3.52 & $H 3 x\left(H 3 x^{\prime}\right)$ & 1.472 & $1.58-1.62$ \\
\hline H3y(H3y') & 4.256 & & H3y(H3y') & 1.930 & \\
\hline H7(H7') & 7.161 & $6.99-7.01$ & $\mathrm{H} 4 \mathrm{x}\left(\mathrm{H} 4 \mathrm{x}^{\prime}\right)$ & 3.079 & 3.44 \\
\hline H8(H8') & 7.519 & 7.25 & H4y(H4y') & 4.215 & \\
\hline H6(H6’) & 8.064 & 7.31 & H8(H8’) & 7.182 & $6.91-6.94$ \\
\hline \multirow[t]{3}{*}{ H4(H4') } & 8.425 & 8.29 & H9(H9') & 7.510 & 7.13 \\
\hline & & & $\mathrm{H} 7\left(\mathrm{H}^{\prime}{ }^{\prime}\right)$ & 8.066 & 7.23 \\
\hline & & & H5(H5’) & 8.466 & 8.21 \\
\hline
\end{tabular}

TMS: Tetrametil silan, Hidrojen Numarası: H Num.

\section{Deneysel ve Teorik Titreşim Frekansları, Spektrumları}

Alınan FTIR spektrumuna göre, $\mathbf{A}$ ve $\mathbf{B}$ için sırasıyla; $3070-2853$ ve $3071-2925 \mathrm{~cm}^{-1}$ aralığında tüm $v(\mathrm{CH})$ titreşimleri (Mihai ve ark., 2009), 1632 ve $1633 \mathrm{~cm}^{-1}$ de $v(\mathrm{C}=\mathrm{N})$ titreşimi (Gabr ve ark., 2015), 1535 ve $1524 \mathrm{~cm}^{-1}$ de $v(\mathrm{C}=\mathrm{C})_{\text {aromatik }}$ titreşimi, 1345 ve $1346 \mathrm{~cm}^{-1}$ 'de $v(\mathrm{C}-\mathrm{S}-\mathrm{C})$ titreşimi, 755 ve $756 \mathrm{~cm}^{-1}$ de tiyofen halkasına ait $v(\mathrm{CS})$ titreşimi gözlendi (Gudasi ve ark., 2006; Mihai ve ark., 2009). Başlangıç maddelerine ait $\sim 3500-3300 \mathrm{~cm}^{-1}$ de primer aminlerin tipik keskin simetrik ve asimetrik $v(\mathrm{NH})$ titreşimleri ve aldehite ait $\sim 1750 \mathrm{~cm}^{-1}$ de $v(\mathrm{C}=\mathrm{O})$ titreşimi ise kayboldu.

A ve B B3LYP teorisi ve $6-311++\mathrm{G}(2 \mathrm{~d}, \mathrm{p})$ temel seti kullanılarak titreşim frekansları hesaplandı. YFT seviyesi gibi kuantum kimyasal metodlarda hesaplanan titreşim frekansları ile deneysel titreşim frekansları arasında küçük sapmalar görüldü. Deneysel ölçümlerdeki harmonik olmayan ve band örtüşme etkilerinden kaynaklan (Hriday ve ark., 2013) bu sapmay1 ortadan kaldırmak için teorik olarak hesaplanan frekanslar 6-311++G(2d,p) temel setine uygun skala faktörü olan 0.9613 ile çarpıldı (Al-Otaibi ve Al-Wabli, 2015; Uluçam ve ark., 2015). Ligantların seçilen bazı düzeltilmiş titreşim frekansları Çizelge VI'da sunuldu.

A için $v \mathrm{C} 4 \mathrm{~N}$ titreşimi $1628 \mathrm{~cm}^{-1}$, de, $v \mathrm{C} 5 \mathrm{SC} 8$ titreşimi $1336 \mathrm{~cm}^{-1}$, de ve $v \mathrm{C} 8 \mathrm{~S}, v \mathrm{C} 5 \mathrm{~S}$ titreşimleri $829,790 \mathrm{~cm}^{-1}$ de ve $\mathbf{B}$ için $v \mathrm{C} 5 \mathrm{~N}$ titreşimi $1628 \mathrm{~cm}^{-1}$ 'de, vC6SC9 titreşimi $1636 \mathrm{~cm}^{-1}$, de ve vC9S, vC6S titreşimleri 828, $777 \mathrm{~cm}^{-1}$, de hesaplandi. Teorik ve deneysel tüm titreşimler karşılaştırıldığında uyumlu olduğu gözlendi. 
Çizelge 6. A ve B ligantlarının deneysel ve teorik (B3LYP, 6-311++G(2d,p)) FTIR değerleri ( $\left.\mathrm{cm}^{-1}\right)$

\begin{tabular}{|c|c|c|c|c|c|c|c|}
\hline \multicolumn{4}{|c|}{ A A } & \multicolumn{4}{|c|}{ B B } \\
\hline $\begin{array}{l}\text { Seç. tit. } \\
\text { fre. }\end{array}$ & $\begin{array}{c}\text { Deneysel } \\
\left(\mathrm{cm}^{-1}\right)\end{array}$ & $\begin{array}{l}\text { Teorik } \\
\left(\mathrm{cm}^{-1}\right)\end{array}$ & $\begin{array}{l}\text { Seç. tit. } \\
\text { fre. }\end{array}$ & $\begin{array}{l}\text { Seç. tit. } \\
\text { fre. }\end{array}$ & $\begin{array}{c}\text { Deneysel } \\
\left(\mathrm{cm}^{-1}\right)\end{array}$ & $\begin{array}{l}\text { Teorik } \\
\left(\mathrm{cm}^{-1}\right)\end{array}$ & $\begin{array}{l}\text { Seç. tit. } \\
\text { fre. }\end{array}$ \\
\hline \multirow{6}{*}{$v \mathrm{CH}$} & \multirow{6}{*}{$\begin{array}{l}3070- \\
2853\end{array}$} & 3120 & $v \mathrm{C} 8 \mathrm{H} 8$ & \multirow{6}{*}{$v \mathrm{CH}$} & \multirow{6}{*}{$\begin{array}{l}3071- \\
2925\end{array}$} & 3119 & $v \mathrm{C} 9 \mathrm{H} 9$ \\
\hline & & 3095 & $\begin{array}{l}v_{\mathrm{s}} \mathrm{C} 6 \mathrm{H} 6 \\
v_{\mathrm{s}} \mathrm{C} 7 \mathrm{H} 7\end{array}$ & & & 3095 & $\begin{array}{l}v_{\mathrm{s}} \mathrm{C} 7 \mathrm{H} 7 \\
v_{\mathrm{s}} \mathrm{C} 8 \mathrm{H} 8\end{array}$ \\
\hline & & 3075 & $\begin{array}{l}v_{\mathrm{as}} \mathrm{C} 6 \mathrm{H6} \\
v_{\mathrm{as}} \mathrm{C} 7 \mathrm{H} 7\end{array}$ & & & 3075 & $\begin{array}{l}v_{\mathrm{as}} \mathrm{C} 7 \mathrm{H} 7 \\
v_{\mathrm{as}} \mathrm{C} 8 \mathrm{H} 8\end{array}$ \\
\hline & & 2945 & $\begin{array}{l}v_{\text {as }} \mathrm{C} 1 \mathrm{H} 1 \mathrm{xH} 1 \mathrm{y} \\
v_{\mathrm{as}} \mathrm{C} 2 \mathrm{H} 2 \mathrm{xH} 2 \mathrm{y}\end{array}$ & & & 2942 & $\begin{array}{l}v_{\mathrm{as}} \mathrm{C} 1 \mathrm{H} 1 \mathrm{xH} 1 \mathrm{y} \\
v_{\mathrm{as}} \mathrm{C} 2 \mathrm{H} 2 \mathrm{xH} 2 \mathrm{y} \\
v_{\mathrm{as}} \mathrm{C} 3 \mathrm{H} 3 \mathrm{xH} 3 \mathrm{y}\end{array}$ \\
\hline & & 2908 & $v_{\mathrm{as}} \mathrm{C} 3 \mathrm{H} 3 \mathrm{xH} 3 \mathrm{y}$ & & & 2908 & $\mathrm{v}_{\mathrm{as}} \mathrm{C} 4 \mathrm{H} 4 \mathrm{xH} 4 \mathrm{y}$ \\
\hline & & 2876 & $v_{\mathrm{s}} \mathrm{C} 1 \mathrm{H} 1 \mathrm{xH} 1 \mathrm{y}$ & & & 2875 & $v_{\mathrm{s}} \mathrm{C} 1 \mathrm{H} 1 \mathrm{xH} 1 \mathrm{y}$ \\
\hline$v \mathrm{CN}$ & 1632 & 1628 & $v \mathrm{C} 4 \mathrm{~N}$ & $v \mathrm{CN}$ & 1633 & 1628 & $v \mathrm{C} 5 \mathrm{~N}$ \\
\hline$v \mathrm{CC}$ & 1535 & 1501 & $\begin{array}{l}\mathrm{vC5C6} \\
v \mathrm{C} 7 \mathrm{C} 8\end{array}$ & $v \mathrm{CC}$ & 1524 & 1501 & $\begin{array}{l}\mathrm{vC7C6} \\
\text { vC8C9 }\end{array}$ \\
\hline$m \mathrm{CH} 2$ & & 1450 & $\begin{array}{l}m \mathrm{C} 1 \mathrm{H} 1 \mathrm{xH} 1 \mathrm{y} \\
m \mathrm{C} 2 \mathrm{H} 2 \mathrm{xH} 2 \mathrm{y}\end{array}$ & $m \mathrm{CH} 2$ & & 1445 & $\begin{array}{l}m \mathrm{C} 1 \mathrm{H} 1 \mathrm{xH} 1 \mathrm{y} \\
m \mathrm{C} 2 \mathrm{H} 2 \mathrm{xH} 2 \mathrm{y} \\
m \mathrm{C} 3 \mathrm{H} 3 \mathrm{xH} 3 \mathrm{y}\end{array}$ \\
\hline$m \mathrm{CH} 2$ & & 1430 & $m \mathrm{C} 3 \mathrm{H} 3 \mathrm{xH} 3 \mathrm{y}$ & $m \mathrm{CH} 2$ & & 1431 & $m \mathrm{C} 4 \mathrm{H} 4 \mathrm{xH} 4 \mathrm{y}$ \\
\hline$v \mathrm{CC}$ & & 1400 & $\begin{array}{l}v \mathrm{C} 6 \mathrm{C} 7 \\
v \mathrm{C} 4 \mathrm{C} 5\end{array}$ & $v \mathrm{CC}$ & & 1400 & $\begin{array}{l}\mathrm{vC7C8} \\
v \mathrm{C} 5 \mathrm{C} 6\end{array}$ \\
\hline$\delta \mathrm{CH}$ & & 1356 & 8C4H4 & $\delta \mathrm{CH}$ & & 1356 & $\delta \mathrm{C} 5 \mathrm{H} 5$ \\
\hline$v \mathrm{CSC}$ & 1345 & 1336 & $v \mathrm{C5SC} 8$ & $v \mathrm{CSC}$ & 1346 & 1336 & $v \mathrm{C6SC} 9 v$ \\
\hline$v \mathrm{CC}$ & & 1119 & $v \mathrm{C} 4 \mathrm{C} 5$ & $v \mathrm{CC}$ & & 1115 & $v \mathrm{C} 5 \mathrm{C} 6$ \\
\hline$\gamma \mathrm{CH}$ & & 946 & $\gamma \mathrm{C} 4 \mathrm{H} 4$ & $\gamma \mathrm{CH}$ & & 947 & $\gamma \mathrm{C} 5 \mathrm{H} 5$ \\
\hline & & 829 & $v \mathrm{C} 8 \mathrm{~S}$ & & & 828 & $\nu \mathrm{C} 9 \mathrm{~S}$ \\
\hline$v \mathrm{CS}$ & 755 & 790 & $v \mathrm{C} 5 \mathrm{~S}$ & $v \mathrm{CS}$ & 756 & 777 & $v \mathrm{C} 6 \mathrm{~S}$ \\
\hline
\end{tabular}

Titreşim modları: $v_{\mathrm{s}}$ : simetrik gerilme, $v_{\mathrm{as}}$ : simetrik gerilme, $\delta$ : düzlem içi sallanma, $\gamma$ : düzlem dış1 sallanma, $m$ : düzlem içi makaslama, $b$ : düzlem dışı burulma. Seçilmiş titreşim frekansları: Seç. tit. fre.

\section{Antibakteriyal Aktivite}

A ve B'nin beş bakteri üzerinde antibakteriyal aktiviteleri araştırıldı. 96 kuyucuklu plakalara ekilen bakteri üzerine madde ve antibiyotik konsantrasyonları ayrı ayrı ilave edildi. $37^{\circ} \mathrm{C}$ de
24 saat inkübasyona birakıldı. İnkübasyon sonunda elisa plaka okuyucuda absorbans değerleri ölçüldü. Değerler \%canlılık olarak hesaplanarak grafiğe aktarıldı (Şekil 5). Hazırlanan altı dozda antibakteriyal aktivite göstermediği belirlendi. 

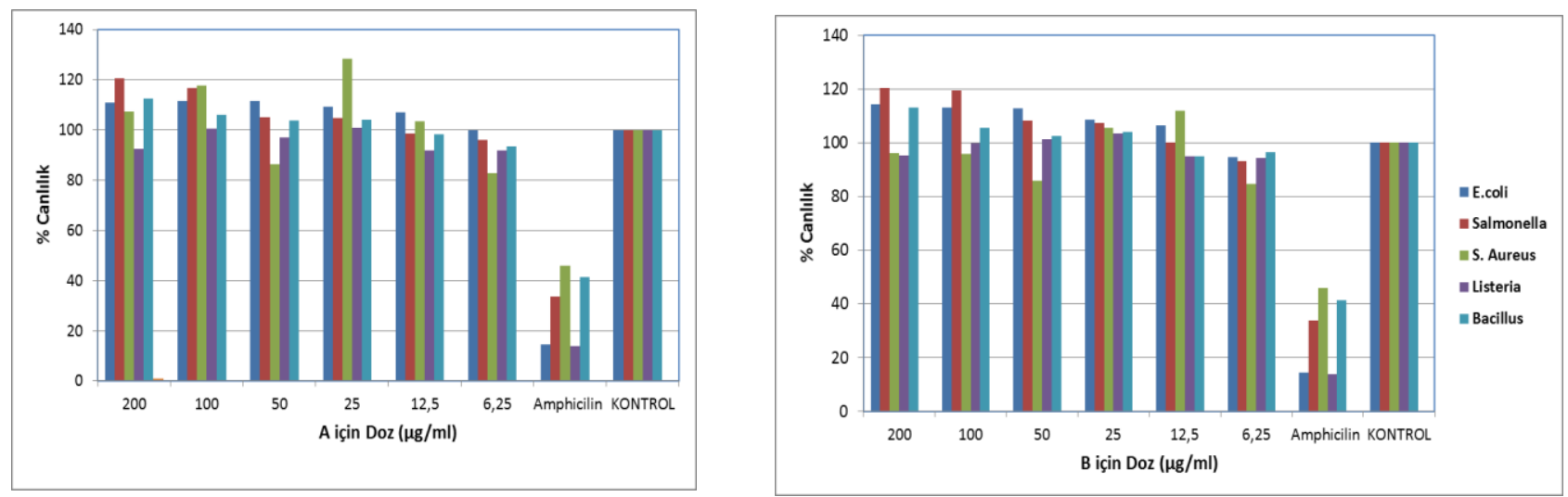

Şekil 5. A ve B ligantlarının antibakteriyal aktivite grafiği

\section{SONUÇ}

2-tiyofen-karboksialdehit ile sirasiyla 1,6diaminohekzan ve 1,8-diaminooktan schiff baz kondenzasyon metodu ile $\mathbf{A}$ ve $\mathbf{B}$ elde edilmiştir. Ligantların yapısı FTIR, ${ }^{1} \mathrm{H}-$ ve ${ }^{13} \mathrm{C}-\mathrm{NMR}, \mathrm{LC}$ ESI/MS ile açıklanmıştır. Ligantlarımız, Escherichia coli ATCC 25922, Staphylococcus aureus ATCC 25923, Listeria monocytogenes ATCC 19115, Salmonella thphimurium ATCC 14028, Bacillus cereus ATCC 11778 bakterileri üzerine altı dozda da kayda değer bir antibakteriyal aktivite göstermemiştir. Ligantların teorik özellikleri gaz fazında B3LYP/6-311G++(2d,p) temel set kullanilarak bağ uzunlukları, bağ ve dihedral açıları bulunmuştur. Ayrıca, Gaussian G09w paket program kullanılarak NMR ve FTIR değerleri hesaplamıştır. Deneysel ve teorik FTIR ve NMR spektrumları da oldukça uyumlu olduğu gözlenmiştir. HOMO ve LUMO enerjileri teorik olarak bulunan değerleri kullanılarak ligantların, elektronegatifliği, kimyasal potansiyeli, kimyasal yumuşaklığı ve sertliği hesaplanmıştır. Ligantların azotlarının bulunduğu kırmızı bölgeden nükleofilik davranış sergileyeceği MEP haritasından saptanmıştır. Deneysel ve teorik spektrum verileri karşılaştırılarak uyumlu olduğu tesbit edilmiş ve molekülün uzayda nasıl bulunduğu hakkında bilgiler ortaya konulmuştur

\section{TEŞEKKÜR}

Çalışma TÜBAP-2016/22 projesi ile Trakya Üniversitesi Araştırma Fonu tarafından desteklenmiştir.

\section{KAYNAKLAR}

Al-Otaibi JS, Al-Wabli RI, 2015. Vibrational spectroscopic investigation (FT-IR and FTRaman) using ab initio (HF) and DFT (B3LYP) calculations of 3-ethoxymethyl-1,4dihydroquinolin-4-one. Spectrochimica Acta Part A: Molecular and Biomolecular Spectroscopy, 137: 7-15.

Alkorta I, Perez JJ, 1996. Molecular polarization potential maps of the nucleic acid bases. International Journal of Quantum Chemistry, 57 (1): 123-135.

Altürk S, Avcı D, Tamer Ö, Atalay Y, 2018. 1Hpyrazole-3-carboxylic acid: Experimental and computational study. Journal of Molecular Structure, 1164: 28-36.

Casella L, Ibers JA, 1981. Synthesis, Characterization, and Reactivity of Copper(I) and Copper(II) Complexes of N,N'-Bis(3-(2Thenylideneimino)Propyl)Piperazine (Tipp) and

$\mathrm{N}, \mathrm{N}$ '-Bis $(3-(2-$

Thenylamino)Propyl)Piperazine (Tapp). Crystal Structure of $[\mathrm{Cu}(\mathrm{Tapp})][\mathrm{Clo} 4] 2$. Inorganic Chemistry, 20 (8): 2438-2448. 
Dineshkumar S, Muthusamy A, 2016. Synthesis and Spectral Characterization of Cross Linked Rigid Structured Schiff Base Polymers: Effect of Substituent Position Changes on Optical, Electrical, and Thermal Properties. PolymerPlastics Technology and Engineering, 55 (4): 368-378.

Fedorova EV, Buryakina AV, Vorobieva NM, Baranova NI, 2013. The Vanadium Compounds: Chemistry, Synthesis, Insulinomimetic Properties. Biochemistry (Moscow) Supplement Series B: Biomedical Chemistry, 7 (4): 259-270.

Gabr MT, El-Gohary NS, El-Bendary ER, ElKerdawy MM, Ni N, Shaaban MI, 2015. Synthesis, antimicrobial, antiquorum-sensing and cytotoxic activities of new series of benzothiazole derivatives. Chinese Chemical Letters, 26 (12): 1522-1528.

Gadre SR, Shrivastava IH, 1991. Shapes and sizes of molecular anions via topographical analysis of electrostatic potential. The Journal of Chemical Physics, 94 (6): 4384-4390.

Gudasi KB, Patil MS, Vadavi RS, Shenoy RV, Patil SA, Nethaji M, 2006. X-ray Crystal Structure of the N-(2-hydroxy-1naphthalidene)phenylglycine Schiff Base. Synthesis and Characterization of its Transition Metal Complexes. Transition Metal Chemistry, 31 (5): 580-585.

Hamada Y, 1997. The development of chelate metal complexes as an organic electroluminescent material. IEEE Transactions on Electron Devices, 44 (8): 1208-1217.

Hriday MN, Srivastava RK, Narayan V, S. C, Sachan AK, Shukla VK, Prasad O, Sinha L, 2013. Electronic structure, non-linear properties and vibrational analysis of ortho, meta and para -hydroxybenzaldehyde by density functional theory. Research Journal of Recent Sciences, 2: 150-157.
Karakaya C, Dede B, Cicek E, 2016. Novel Metal(II) Complexes with Bidentate Schiff Base Ligand: Synthesis, Spectroscopic Properties and Dye Decolorization Functions. Acta Physica Polonica A, 129 (2): 208-212.

Kumar S, Nath Dhar D, N. Saxena P, 2009. Applications of metal complexes of Schiff bases-A review. Journal of scientific and industrial research, 68 (3): 181-187.

Luque FJ, Orozco M, Bhadane PK, Gadre SR, 1993. SCRF calculation of the effect of water on the topology of the molecular electrostatic potential. The Journal of Physical Chemistry, 97 (37): 9380-9384.

Mihai S, Negoiu M, Bondarev A, 2009. Synthesis, Characterization and Biological Activity of Some Novel Metal Complexes of Schiff Base Derived from p-phenyldiamine and 2thiophene Carboxaldehyde. Revista de Chimie -Bucharest, 60: 778-782.

Murray JS, Sen K. 1996. Molecular Electrostatic Potentials, Concepts and Applications, Elsevier Science, No: 1, pp. 665, AmsterdamNetherlands

Nagy PI, 2013. The syn-anti equilibrium for the $\mathrm{COOH}$ group reinvestigated. Theoretical conformation analysis for acetic acid in the gas phase and in solution. Computational and Theoretical Chemistry, 1022: 59-69.

Pessoa JC, Etcheverry S, Gambino D, 2015. Vanadium Compounds in Medicine. Coordination Chemistry Reviews, 301-302 (Supplement C): 24-48.

Rehder D, 2003. Biological and Medicinal Aspects of Vanadium. Inorganic Chemistry Communications, 6 (5): 604-617.

Sreedasyam JS, Sunkari J, Kundha S, Gundapaneni RR, 2013. N,N'-(Propane-1,3-diyl)bis(2aminobenzamide). Acta Crystallographica Section E, 69 (5): 0673. 
Sun XL, Fan LX, Yang YJ, Guo Z, Tian WQ, Lei S, 2015. Synthesis of one-dimensional Schiff base polymers that contain an oligothiophene building block on the graphite surface. Chemistry, 21 (18): 6898-6905.

Suresh T, Shivaraj, Durgad K, Suresh, 2015. Synthesis Characterization and Biological Studies on Drug Metal Complexes. International Journal of Pharmacy \& Life Sciences, 6 (8/9): 4719-4726.

Tamer Ö, Avc1 D, Atalay Y, 2016. Synthesis, XRay crystal structure, photophysical characterization and nonlinear optical properties of the unique manganese complex with picolinate and 1,10 phenantroline: toward the designing of new high NLO response crystal. Journal of Physics and Chemistry of Solids, 99: 124-133.

Thompson KH, 1999. Vanadium and diabetes. Biofactors, 10 (1): 43-51.

Turkyilmaz M, Uluçam G, Aktaş Ş, Okan SE, 2017. Synthesis and Characterization of New N-Heterocyclic Carbene Ligands: 1,3Bis(Acetamide)Imidazol-3-Ium Bromide And 3-(Acetamide)-1-(3-Aminopropyl)-1hImidazol-3-Ium Bromide. Journal of Molecular Structure, 1136 (Supplement C): 263-270.
Ulucam G, 2017. Synthesis, Characterization and Biological Activities of 3-(2-Hydroxyethyl)-1(3-Aminopropyl)-1HImidazole-3-Ium Bromide. Trakya University Journal of Natural Sciences, 18 (1): 49-54.

Ulucam G, Turkyilmaz M, 2018. Synthesis, Structural Analysis, and Biological Activities of Some Imidazolium Salts. Bioinorganic chemistry and applications, 2018: 1439810.

Uluçam G, Okan SE, Aktaş Ş, Öğretmen GP, 2015. Characterization of dinaphthosulfoxide molecule. Journal of Molecular Structure, 1102: 146-152.

Vela A, Gazquez JL, 1990. A relationship between the static dipole polarizability, the global softness, and the fukui function. Journal of the American Chemical Society, 112 (4): 14901492. 